\title{
High-mass microquasars and low-latitude gamma-ray sources
}

\author{
V. Bosch-Ramon ${ }^{1}$, G. E. Romero ${ }^{2,3, \star}$, and J. M. Paredes ${ }^{1}$ \\ ${ }^{1}$ Departament d'Astronomia i Meteorologia, Universitat de Barcelona, Av. Diagonal 647, 08028 Barcelona, Spain \\ e-mail: vbosch@am.ub.es; jmparedes@ub.edu \\ 2 Instituto Argentino de Radioastronomía, C.C.5, (1894) Villa Elisa, Buenos Aires, Argentina \\ e-mail: romero@iar.unlp.edu.ar \\ 3 Facultad de Ciencias Astronómicas y Geofísicas, UNLP, Paseo del Bosque, 1900 La Plata, Argentina
}

Received 29 April 2004 / Accepted 11 August 2004

\begin{abstract}
Population studies of unidentified EGRET sources suggest that there exist at least three different populations of galactic gamma-ray sources. One of these populations is formed by young objects distributed along the galactic plane with a strong concentration toward the inner spiral arms of the Galaxy. Variability, spectral and correlation analysis indicate that this population is not homogeneous. In particular, there is a subgroup of sources that display clear variability in their gammaray fluxes on timescales from days to months. Following the proposal by Kaufman Bernadó et al. (2002), we suggest that this group of sources might be high-mass microquasars, i.e. accreting black holes or neutron stars with relativistic jets and early-type stellar companions. We present detailed inhomogeneous models for the gamma-ray emission of these systems that include both external and synchrotron self-Compton interactions. We have included effects of interactions between the jet and all external photon fields to which it is exposed: companion star, accretion disk, and hot corona. We make broadband calculations to predict the spectral energy distribution of the emission produced in the inner jet of these objects up to GeV energies. The results and predictions can be tested by present and future gamma-ray instruments like INTEGRAL, AGILE, and GLAST.
\end{abstract}

Key words. X-rays: binaries - stars: winds, outflows - gamma-rays: observations - gamma-rays: theory

\section{Introduction}

Population studies of the unidentified gamma-ray sources detected by the EGRET instrument of the Compton Gamma-Ray Observatory (3rd EGRET catalog, Hartman et al. 1999) clearly suggest the existence of at least three different groups of galactic sources (Gehrels et al. 2000; Grenier 2001, 2004; Romero 2001). There is a group of weak and steady sources positionally correlated with the Gould Belt (Grenier 1995; Gehrels et al. 2000). These sources are thought to be nearby (100-300 pc), with isotropic luminosities $\sim 10^{33} \mathrm{erg} \mathrm{s}^{-1}$. Following Romero et al. (2004), we will call these sources the Local Gamma-Ray Population (LGRP). There are $45 \pm 5$ sources in this group.

Another group of sources is concentrated along the Galactic Plane. They are well-correlated with star forming regions and HII regions, which is indicative of an association with young stellar objects (Romero et al. 1999; Romero 2001). $\log N-\log S$ studies suggest that they are more abundant toward the inner spiral arms (Gehrels et al. 2000; Bhattacharya et al. 2003). These are bright sources (isotropic luminosities in the range $10^{34-36} \mathrm{erg} \mathrm{s}^{-1}$ ), with an average photon spectral index $\Gamma=2.18 \pm 0.04\left(F(E) \propto E^{-\Gamma}\right)$. These sources, whose

^ Member of CONICET. number is $\sim 45 \pm 9$, form the Gamma-Ray Population I (GRP I, Romero et al. 2004).

Finally, there is a third group of sources that are distributed forming a kind of halo around the galactic center. These sources have higher luminosities, in the range $10^{34-37} \mathrm{erg} \mathrm{s}^{-1}$. They have soft spectra $(\Gamma \sim 2.5)$ and display strong variability (Nolan et al. 2003). These sources should be old, with ages from hundreds of Myr to Gyr. About $45 \pm 5$ EGRET detections fall in this category (Grenier 2001). They form the Gamma-Ray Population II (GRP II).

Among GRP I sources there is a subgroup that displays significant variability on timescales of weeks to months (Torres et al. 2001; Nolan et al. 2003). Recently, Kaufman Bernadó et al. (2002) and Romero et al. (2004) have suggested that this subgroup of GRP I sources might consist of high-mass microquasars (i.e. microquasars formed by a compact object and an early-type stellar companion), where the gamma-ray emission arises from interactions between relativistic particles in the jet and external photon fields, most notably the stellar UV emission. Population studies by several authors (Grimm et al. 2002; Miyagi \& Bhattacharya 2004) suggest that high-mass X-ray binaries (HMXB) are distributed in the Galaxy following the spiral structure, presenting a steeper $\log N-\log S$ distribution than low-mass X-ray binaries (LMXB), which means 
that LMXB are more uniformly distributed in the Galaxy than HMXB. Since high-mass microquasars form a subset of the whole set of HMXB, they are also expected to be distributed along the spiral arms, as is the case of GRP I sources.

In the present paper we explore in more detail this hypothesis, presenting more realistic models for the gamma-ray emission. In particular, we will include effects of the interaction of the microquasar jet with the $\mathrm{X}$-ray fields produced by the accretion disk and the hot corona that is thought to surround the compact object. We will also include synchrotron self-Compton emission, Klein-Nishina effects, and the backreaction of the different losses in the particle spectrum of the jet. We will calculate, for some representative sets of the parameters that characterize high-mass microquasars, the broadband spectral energy distribution (SED) of these objects.

We will start discussing the main phenomenological properties of GRP I sources in order to clarify what we should expect from our models.

\section{GRP I sources}

GRP I sources concentrate along the galactic plane and present a good spatial correlation with young stellar objects (Romero et al. 1999). The variability analysis of these sources by Torres et al. (2001) clearly shows evidence for the existence of a subgroup with variable emission on timescales from weeks to months. This is corroborated by the recent results presented by Nolan et al. (2003), which are based on a maximum likelihood re-analysis of the EGRET data. These authors identify 17 variable sources within $\left|6^{\circ}\right|$ from the galactic plane. These sources are clumped within $\left|55^{\circ}\right|$ of the galactic center.

A $\log N-\log S$ analysis for all GRP I sources yields a distribution that is consistent with a power-law with index $\beta \sim 3.1$ (Bhattacharya et al. 2003). This is far steeper than what is expected for a population uniformly distributed along the galactic disk. For instance, for pulsars detected at $400 \mathrm{MHz}$ the slope is $\beta \sim 1.05$. The unidentified gamma-ray sources, on the contrary, seem to be concentrated mainly in the inner spiral arms. To find possible further evidence for different populations among GRP I sources, we have implemented a $\log N-\log S$ analysis of both variable and non-variable low-latitude sources.

First we have considered the 17 variable sources listed by Nolan et al. (2003). To take into account systematic effects introduced by different exposure and background resulting in non-uniform detectability, we have adopted the procedure described by Reimer (2001). The obtained $\log N-\log S$ plot is shown in Fig. 1, lower panel. The normalized distribution can be fitted by a power-law $N(S) \propto S^{-\beta}$, with $\beta=1.66 \pm 0.31$, significantly harder than for the entire sample. If we now consider those sources that classify as non-variable or dubious cases, we get the $\log N-\log S$ plot shown also in Fig. 1, upper panel. In this case the distribution can be fitted by a power-law with index $\beta=2.92 \pm 0.36$.

The average spectral index is also different for both samples: in the case of the variable sources we have $\langle\Gamma\rangle=2.04 \pm$ 0.03 , whereas for the remaining sources $\langle\Gamma\rangle=2.16 \pm 0.01$. All this suggests that there are two different groups of sources, one formed by steady sources concentrated toward the inner spiral

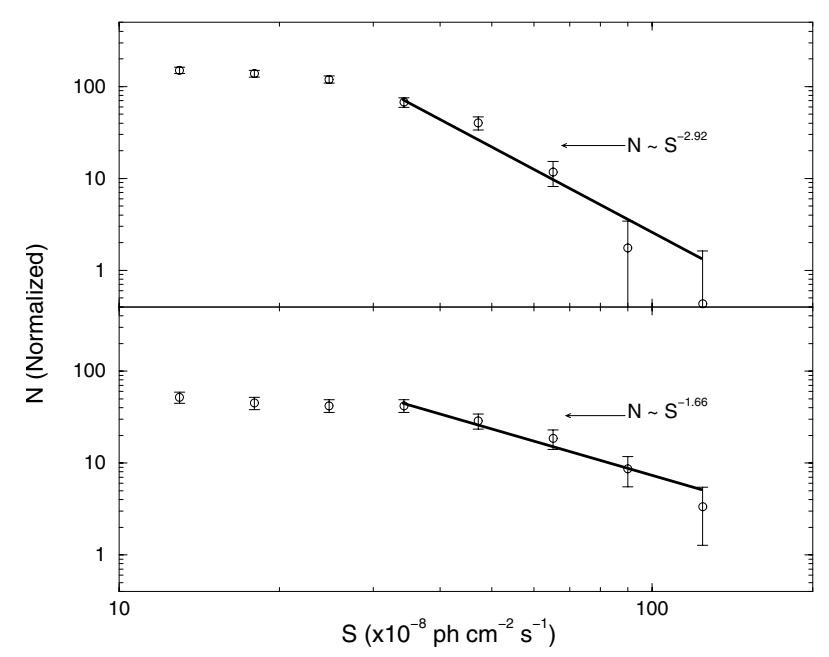

Fig. 1. $\log N-\log S$ plot for gamma-ray sources within $|b|<6^{\circ}$. a) Upper panel: For non variable gamma-ray sources. b) Lower panel: For variable gamma-ray sources.

arms, and a second group with variable sources and a wider distribution along the galactic plane, although not as wide as that of radio pulsars (Bhattacharya et al. 2003).

Microquasars appear to be good candidates for compact and variable sources in the galactic plane. Since they can have very large proper motions (e.g. Ribó et al. 2002; Mirabel et al. 2004), their distribution along the plane should be broader than that presented by supernova remnants and molecular clouds, which can be traced by star-forming regions and OB associations. Their spread, however, is limited by the lifespan of the companion massive star, and hence it is not as extended as that of radio pulsars. It is worth noting that Miyagi \& Bhattacharya (2004) obtained for HMXB a $\beta$ of about 1.9. In the next section we will discuss the potential of microquasars to be gamma-ray sources.

\section{Gamma-ray emission from microquasars}

Microquasars are variable sources at all wavelengths where they have been detected. At radio frequencies they present non-thermal jets and in some cases superluminal components (Mirabel \& Rodríguez 1999). In the case of high-mass microquasars, with which we are concerned here, the emission at optical and UV energies is dominated by the companion star, where it can reach luminosities of $\sim 10^{39} \mathrm{erg} \mathrm{s}^{-1}$. Accretion onto the compact object results in the formation of an accretion disk with typical temperatures of a few $\mathrm{keV}$ and thermal luminosities that can reach $\sim 10^{37} \mathrm{erg} \mathrm{s}^{-1}$. In the low-hard state, when the jets appear to be stronger, microquasars usually present a hard X-ray component that can be represented by a power-law plus an exponential cutoff at a few hundred keV. This component might be produced by a hot corona or ADAF region around the central object (e.g. Poutanen 1998; McClintock \& Remillard 2004) although some authors have argued for a purely non-thermal origin in the jets (Markoff et al. 2001, 2003; Georganopoulos et al. 2002). Beyond the problem of the exact nature of the different contributions to the hard X-rays, it is clear that very relativistic electrons are present in the jets of 
microquasars. Since these electrons must traverse several photon fields, inverse Compton interactions are unavoidable. Such interactions can produce high-energy gamma-rays with a luminosity that will depend on the particular physical parameters that characterize the source.

In recent years, several authors have studied models for leptonic gamma-ray production in microquasars (Atoyan \& Aharonian 1999; Paredes et al. 2000, 2002; Kaufman Bernadó et al. 2002; Romero et al. 2002; Bosch-Ramon \& Paredes 2004a). Most of these models consider steady and compact jets. Such jets are usually associated with the low-hard X-ray state of the sources. However, some microquasars present persistent radio jets and moderate X-ray emission. This is the case of the two already known microquasars that have been proposed to be counterparts of EGRET sources: LS 5039 (Paredes et al. 2000, 2002) and LS I +61 303 (Massi et al. 2004; Bosch-Ramon \& Paredes 2004b). The fact that a new microquasar candidate with similar X-ray properties has been recently discovered by Combi et al. (2004) within the EGRET location error box of 3EG J1639-4702, lends additional support to the idea that perhaps high-energy gamma-ray emitting microquasars are not typical X-ray binary systems from the point of view of their X-ray behavior.

In the present paper we will consider a microquasar with a continuous, persistent and inhomogeneous jet endowed with a structure similar to that adopted by Romero et al. (2003). In our case, however, we will focus on the leptonic content. The jet is ejected along the rotation axis of the compact object, which for simplicity is assumed to be perpendicular to the orbital plane ${ }^{1}$.

The relativistic jet flow will move along the $z$ axis with a bulk Lorentz factor $\Gamma_{\text {jet }}$ and a constant velocity $\beta=v / c$. Hadrons, although carrying most of the kinetic power, will play no radiative role in our model (for hadronic gamma-ray emission see the paper by Romero et al. 2003). We shall allow the jet to expand laterally, in such a way that the radius $R$ at a distance $z$ from the compact object will be given by $R(z)=\xi z^{\varepsilon}$, with $\varepsilon \leq 1$ and $z_{0} \leq z \leq z_{\max }$. For $\varepsilon=1$ we have a conical jet.

The relativistic electrons in the jet are assumed to have an energy distribution given by a power-law, as inferred from the observed synchrotron emission:

$N\left(z, \gamma_{\mathrm{e}}\right)=k(z) \gamma_{\mathrm{e}}^{-p}$

$k(z)=k\left(z_{0}\right)\left(\frac{R_{0}}{R}\right)^{2}=k\left(z_{0}\right)\left(\frac{z_{0}}{z}\right)^{2 \varepsilon}$.

Here, $p$ is the power-law index of the electron energy distribution and $R_{0}=R\left(z_{0}\right)$ is the radius of the jet at the injection point. $\gamma_{\mathrm{e}}$ is the electron Lorentz factor and $N\left(z, \gamma_{\mathrm{e}}\right)$ is the number of electrons of a given energy per unit of volume at a given distance from the compact object. The electron distribution does not depend on the direction of the particle velocity.

\footnotetext{
${ }^{1}$ Misaligned jets are probably a common case but our results will not be significantly affected by a small inclination angle. See Maccarone (2002).
}

Table 1. Constants in synchrotron formulae.

\begin{tabular}{lc}
\hline \hline Parameter (symbol) & Value \\
\hline$C_{1}$ & $6.27 \times 10^{18} \mathrm{cgs}$ units \\
$C_{5}(p=2)$ & $1.37 \times 10^{-23} \mathrm{cgs}$ units \\
$C_{6}(p=2)$ & $8.61 \times 10^{-41} \mathrm{cgs}$ units \\
\hline
\end{tabular}

Another important parameter associated with the electron energy distribution is the maximum Lorentz factor, $\gamma_{\mathrm{emax}}$. Its evolution is described by:

$\gamma_{\mathrm{emax}}(z)=\gamma_{\mathrm{emax}}\left(z_{0}\right)\left(\frac{z_{0}}{z}\right)^{e \varepsilon}$

The parameter $e$ is introduced to take into account loss or gain energy processes other than adiabatic losses, which are already counted through $\varepsilon$, without a deep description of the involved physical processes (see, i.e., Ghisellini et al. 1985). Both $\varepsilon$ and $e$ cover our lack of knowledge of what happens to/within the jet. In the present work, we will set $e$ to 1 from now on, to simplify the number of free parameters, assuming as a first approximation a jet controlled by adiabatic evolution. We will come back to this issue in Sect. 5.1, analysing more carefully this question.

Depending on the geometrical nature of the flow and assuming adiabatic expansion, the magnetic field changes with $z$ as:

$B(z)=B\left(z_{0}\right)\left(\frac{R_{0}}{R}\right)=B\left(z_{0}\right)\left(\frac{z_{0}}{z}\right)^{\varepsilon}$.

Then, the synchrotron radiation density can be estimated as:

$$
\begin{aligned}
U_{\text {syn }, v}(z) & \approx \frac{s_{v}(z)}{c} \tau_{v}(z) \\
& =\frac{C_{5}}{c C_{6}} B(z)^{-1 / 2}\left(\frac{v}{2 C_{1}}\right)^{5 / 2}\left(\frac{v}{v_{1}(z)}\right)^{-(p+4) / 2},
\end{aligned}
$$

where $s_{v}$ is the source function of the synchrotron emission from an isotropic particle distribution and $\tau_{v}$ is the synchrotron optical depth of the jet. The explicit expressions for both $s_{v}$ and $\tau_{v}$ can be found in Pacholczyk (1970). We use the formulae in the optically thin case because the synchrotron emission mainly comes from frequencies beyond the self-absorption frequency, which is given by

$v_{1}(z) \approx 2 C_{1}\left[R(z) C_{6} k(z)\left(m_{\mathrm{e}} c^{2}\right)^{(p-1)}\right]^{\frac{2}{p+4}} B(z)^{\frac{p+2}{p+4}}$,

where $C_{1}, C_{5}$ and $C_{6}$ are numerical constants given in Table 1. This is the local approximation to the synchrotron radiation field (Ghisellini et al. 1985).

The total radiation field to which the leptons are exposed in the jet also will have a contribution from external sources. These contributions can be modeled as two black body components, one peaked at UV energies (the companion star field) and other at energies $k T \sim 1 \mathrm{keV}$ (the inner accretion disk field), plus a power-law with an exponential cutoff at $k T \sim$ $150 \mathrm{keV}$ (the corona). With the exception of the disk, these contributions are assumed to be isotropic (Romero et al. 2002; Georganopoulos et al. 2002). 
Once $N\left(z, \gamma_{\mathrm{e}}\right)$ and the total energy density in seed photons $\left(U_{\text {total }}\left(\epsilon_{0}, z\right)\right)$ have been determined, the IC interaction between them can be studied calculating the IC spectral energy distribution per energy unit. The cross section for both the Thomson and the Klein-Nishina regimes has been approximated by Blumenthal \& Gould (1970):

$\frac{\mathrm{d} \sigma\left(x, \epsilon_{0}, \gamma_{\mathrm{e}}\right)}{\mathrm{d} \epsilon}=\frac{3 \sigma_{\mathrm{T}} c}{4 \epsilon_{0} \gamma_{\mathrm{e}}^{2}} f(x)$,

where

$$
\begin{aligned}
& f(x)= {\left[2 x \ln x+x+1-2 x^{2}+\frac{\left(4 \epsilon_{0} \gamma_{\mathrm{e}} x\right)^{2}}{2\left(1+4 \epsilon_{0} \gamma_{\mathrm{e}} x\right)}(1-x)\right] } \\
& \times P\left(1 / 4 \gamma_{\mathrm{e}}^{2}, 1, x\right), \\
& x=\frac{\epsilon}{4 \epsilon_{0} \gamma_{\mathrm{e}}^{2}\left(1-\frac{\epsilon}{\gamma_{\mathrm{e}}}\right)}
\end{aligned}
$$

$\epsilon_{0}$ and $\epsilon$ are the energies of the incoming and the outgoing photons, respectively, $\sigma_{\mathrm{T}}$ is the Thomson cross section, and

$P\left(1 / 4 \gamma_{\mathrm{e}}^{2}, 1, x\right)=1$, for $1 / 4 \gamma_{\mathrm{e}}^{2} \leq x \leq 1$,

and 0 otherwise.

The spectral energy distribution for the optically thin case in the jet's reference frame is:

$$
\begin{aligned}
\epsilon L_{\epsilon}= & \epsilon \int_{z_{\min }}^{z_{\max }} \int_{\epsilon_{0 \min }(z)}^{\epsilon_{\max }(z)} \int_{\gamma_{\mathrm{emin}}(z)}^{\gamma_{\mathrm{emax}}(z)} \Sigma(z) U_{\mathrm{tot}}\left(\epsilon_{0}, z\right) \\
& \times N\left(\gamma_{\mathrm{e}}, z\right) \frac{d \sigma\left(x, \epsilon_{0}, \gamma_{\mathrm{e}}\right)}{d \epsilon} \frac{\epsilon}{\epsilon_{0}} \mathrm{~d} \gamma \mathrm{d} \epsilon_{0} \mathrm{~d} z,
\end{aligned}
$$

where $\Sigma(z)$ is the surface of a perpendicular jet slice located at $z$.

Notice that the external fields contributing to $U_{\text {tot }}\left(\epsilon_{0}, z\right)$ should be transformed to the co-moving frame. Detailed expressions for such transformations are given by Dermer \& Schlickeiser (2002). In the observer's reference frame we have:

$\epsilon^{\prime} L_{\epsilon^{\prime}}^{\prime}=D^{2+p} \epsilon^{\prime} L_{\epsilon^{\prime}}$

The integration is performed in the co-moving system and then the result is transformed to the observer's frame, hence the factor $D^{2+p}$, which is the Doppler boosting for a continuous jet. The energy of the scattered photon in the jet's reference frame $(\epsilon)$ is boosted to $\epsilon^{\prime}=D \epsilon$. The Doppler factor $D$ for the approaching jet is given by

$$
\frac{1}{\Gamma_{\mathrm{jet}}(1-\beta \cos \theta)}
$$

where $\beta$ is the velocity of the jet in speed of light units and $\theta$ is the angle between the jet and the line of sight. We note that $P\left(1 / 4 \gamma_{\mathrm{e}}^{2}, 1, x\right)$ restricts the range of $x$ to physical values, where $\epsilon$ cannot be lower than $\epsilon_{0}\left(x=1 / 4 \gamma_{\mathrm{e}}^{2}\right)$ or larger than $\epsilon_{\max }$ $(x=1)$.

In the case of the IC interactions with disk photons, a factor $(1-\cos \theta)^{(p+1) / 2}$ must be introduced in Eq. (11) to take into account the fact that the photons come from behind the jet (Dermer et al. 1992).
To make any calculation of the IC emission of a given microquasar, we have to specify first the jet power in leptons and hence the constant $k$ in Eq. (1). In this work we shall adopt the disk/jet coupling hypothesis formulated by Falcke \& Biermann $(1995,1999)$, i.e. the jet power is proportional to the accretion rate:

$L_{\text {jet }}^{\text {lep }}=\pi R^{2} c \Gamma_{\text {jet }} \beta \int m_{\mathrm{e}} c^{2} k\left(z_{0}\right) \gamma^{1-p} \mathrm{~d} \gamma=q_{\mathrm{e}} L_{\mathrm{acc}}$.

Here, $L_{\mathrm{acc}}=M_{\mathrm{acc}} c^{2}$ is the accretion power onto the compact object and $q_{\mathrm{e}}$ is a number $<1$. The total jet power is $L_{\text {jet }}=$ $q_{\text {tot }} L_{\text {acc }}=\left(q_{\text {hadrons }}+q_{\mathrm{e}}\right) L_{\text {acc }}$. If the hadrons are also relativistic, $q_{\text {hadrons }} \sim q_{\text {tot }} \gg q_{\mathrm{e}}$. Typically, $q_{\mathrm{tot}} \sim 10^{-1}-10^{-3}$ and then $q_{\mathrm{e}}$ is, in the kind of jet we are considering, in the range $\sim 10^{-3}-10^{-5}$.

\section{Specific assumptions}

We make a number of specific assumptions regarding the characterization of the high-mass microquasar model adopted in the calculations of the SED. In particular, we will consider a system where the compact object is a black hole of $10 M_{\odot}$ that accretes $10^{-8} M_{\odot} \mathrm{yr}^{-1}$. The gravitational radius $R_{\mathrm{g}}$ for such an object is $\sim 1.5 \times 10^{6} \mathrm{~cm}$. The companion star has a radius of $R_{*}=15 R_{\odot}$ and a bolometric luminosity of $\sim 5 \times 10^{38} \mathrm{erg} \mathrm{s}^{-1}$. Its radiation field is well represented by a black body with $k T \sim 10 \mathrm{eV}$. The orbital radius is $R_{\text {orb }} \sim 1 \mathrm{AU}$.

Around the black hole there is a thermal disk with typical temperatures given by $k T \sim 1 \mathrm{keV}$. The total disk luminosity is $\sim 10^{37} \mathrm{erg} \mathrm{s}^{-1}$. The hot corona above the central disk is represented by a power law with a hard photon index 1.6 and an exponential cutoff at $\sim 150 \mathrm{keV}$.

Regarding the jet, we assume it is injected at $z_{0} \sim 50 R_{\mathrm{g}}$. Its initial radius is $R_{0} \sim 0.1 z_{0}$ and its lateral expansion is characterized by a coefficient $\varepsilon=1$. The electron energy distribution has a power law index $p=2$. The high-energy cutoff is assumed at $\gamma_{\mathrm{emax}}\left(z_{0}\right)=10^{4}$, whereas the minimum Lorentz factor is $\gamma_{\mathrm{emin}} \sim 1$.

The magnetic field outside the coronal region, $B\left(z_{0}\right)$, is unknown. Most models for jet production require high magnetic fields, but these fields are usually attached to the inner accretion disk. Here we will assume a set of different possible values, in the range $0.1-200 \mathrm{G}$. These values are below equipartition, which could be reached further in the jet (e.g. in the radio emitting region).

All these assumptions are summarized in Table 2.

\section{Calculations}

We have calculated the high-energy SED for a variety of cases using the typical values of the parameters given in Table 2, and different combinations of magnetic field $B\left(z_{0}\right)$, luminosity of the corona $\left(L_{\mathrm{cor}}\right)$, bulk Lorentz factor $\Gamma_{\text {jet }}$ and disk/jet coupling parameter $q_{\mathrm{e}}$. In Table 3 we give the range of these parameters adopted in our calculations.

In Figs. 2 to 5, we show some representative results for the SED produced by the IC up-scattering of stellar, disk, corona, and synchrotron photons, respectively. Curves obtained from different combinations of the parameters are shown in each panel. 
Table 2. Fixed parameters in the models.

\begin{tabular}{lc}
\hline \hline Parameter (symbol) & Value \\
\hline Black hole mass $\left(M_{\mathrm{bh}}\right)$ & $10 M_{\odot}$ \\
Gravitational radius $\left(R_{\mathrm{g}}\right)$ & $1.48 \times 10^{6} \mathrm{~cm}$ \\
Accretion luminosity $\left(L_{\mathrm{acc}}\right)$ & $10^{-8} M_{\odot} c^{2} \mathrm{year}^{-1}$ \\
Stellar radius $\left(R_{*}\right)$ & $15 R_{\odot}$ \\
Stellar bolometric luminosity $\left(L_{*}\right)$ & $5 \times 10^{38} \mathrm{erg} \mathrm{s}^{-1}$ \\
Viewing angle to jet's axis $\theta$ & $10^{\circ}$ \\
Distance from jet's apex to the compact object $\left(z_{0}\right)$ & $50 R_{\mathrm{g}}$ \\
Initial jet radius $\left(R_{0}\right)$ & $0.1 z_{0}$ \\
Orbital radius $\left(R_{\text {orb }}\right)$ & $\sim 1 \mathrm{AU}$ \\
Peak energy of the disk $\left(k T_{\text {disk }}\right)$ & $1 \mathrm{keV}$ \\
Peak energy of the corona & $150 \mathrm{keV}$ \\
Peak energy of the star $\left(k T_{\text {star }}\right)$ & $10 \mathrm{eV}$ \\
Expansion coefficient of the jet $(\varepsilon)$ & 1 \\
Minimum Lorentz factor for electrons in the jet $($ jet frame $)\left(\gamma_{\mathrm{emin}}\right)$ & $\sim 1$ \\
Maximum Lorentz factor for electrons in the jet $($ jet frame $)\left(\gamma_{\mathrm{emax}}\right)$ & $10^{4}$ \\
Electron energy distribution power-law index $(p)$ & 2 \\
Photon index for the corona $\left(\Gamma_{\text {cor }}\right)$ & 1.6 \\
Total disk luminosity $\left(L_{\mathrm{disk}}\right)$ & $10^{37} \mathrm{erg} \mathrm{s}^{-1}$ \\
\hline
\end{tabular}

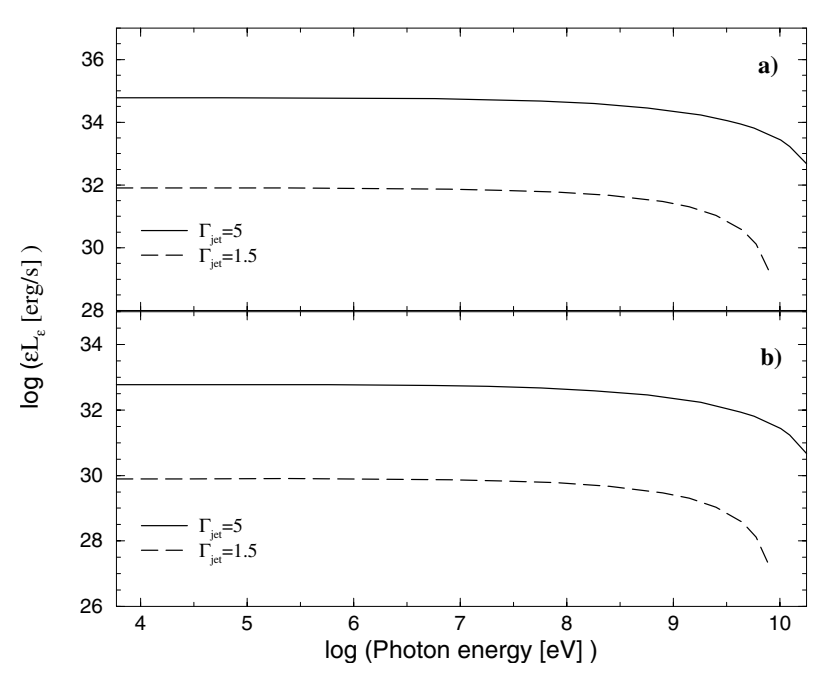

Fig. 2. High-energy SED from IC up-scattering of stellar photons. a) Models with $q_{\mathrm{e}}=10^{-3}$ : for $\Gamma_{\text {jet }}=1.5$ and 5 . b) Models with $q_{\mathrm{e}}=10^{-5}:$ for $\Gamma_{\text {jet }}=1.5$ and 5 .

In Fig. 6, we show the whole SED of a representative case, with the parameters listed in Table 4 . We plot here the spectra for all contributions to the emission: the seed photon sources and the IC components. In Fig. 7 we show a more extreme case, for a source with a high bulk Lorentz factor $\Gamma_{\text {jet }}=10$, a high-energy cutoff for electrons of $\gamma_{\mathrm{emax}}\left(z_{0}\right)=10^{6}$, and a small viewing angle $\theta=1^{\circ}$ (see Table 5). This would correspond to a microblazar, which is also a strong non-thermal X-ray source. It is difficult to say whether persistent microquasar jets could reach such high Lorentz factors, although it is an interesting case to explore.

Finally, we present a "realistic" case, reproducing roughly the SED observed in the two EGRET sources that might be

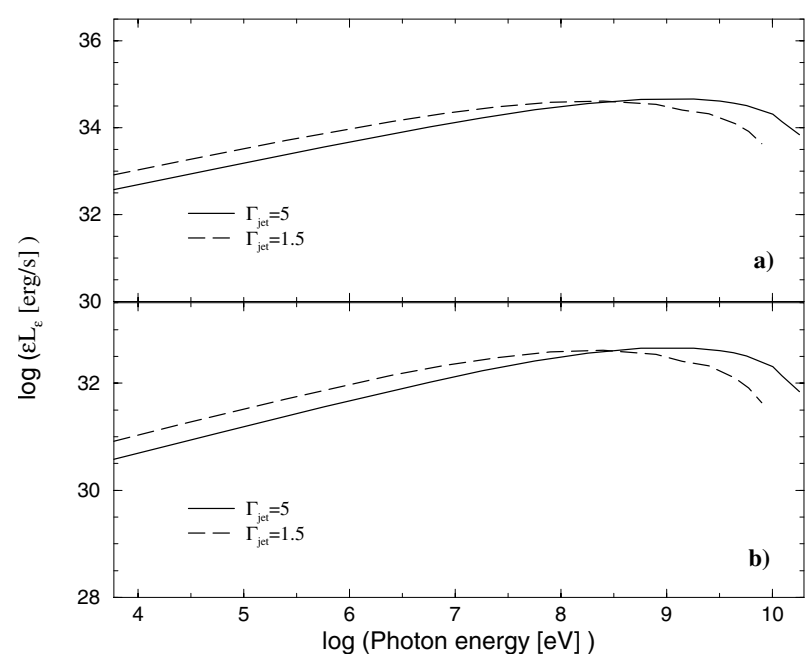

Fig. 3. High-energy SED from IC up-scattering of disk photons. a) Models with $q_{\mathrm{e}}=10^{-3}$ : for $\Gamma_{\text {jet }}=1.5$ and 5 . b) Models with $q_{\mathrm{e}}=10^{-5}:$ for $\Gamma_{\text {jet }}=1.5$ and 5 .

associated with known microquasars: LS 5039/3EG J18241514 and LS I +61 303/3EG J0241+6103 (see Fig. 8 and Table 6). For this particular case, we have taken both the disk and the corona to be faint, as it appears to be the case in both sources, with $L_{\mathrm{cor}}=3 \times 10^{32} \mathrm{erg} \mathrm{s}^{-1}$. Also, the magnetic field required now to match the observations is $\sim 200 \mathrm{G}, q_{\mathrm{e}}$ is $\sim 10^{-3}$, and consistent with observational constraints from both sources, a mildly relativistic jet velocity $\left(\Gamma_{\text {jet }}=1.1\right)$ is used. The electron high-energy cutoff is at $\gamma_{\mathrm{emax}}\left(z_{0}\right) \sim 10^{4}$, in agreement with EGRET data (see Fig. 8). 


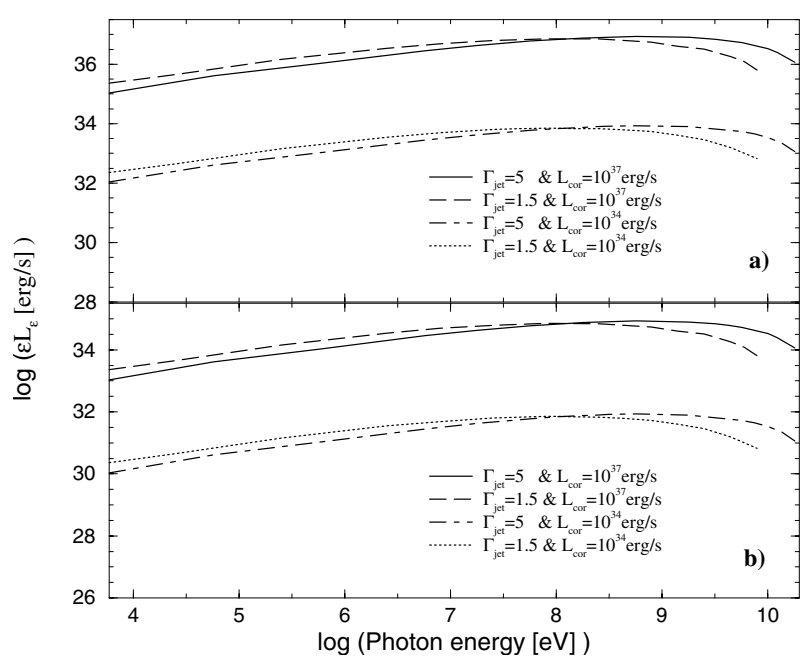

Fig. 4. High-energy SED from IC up-scattering of corona photons. a) Models with $q_{\mathrm{e}}=10^{-3}$ : for $\Gamma_{\text {jet }}=1.5,5$; and $L_{\text {cor }}=10^{34}$, $10^{37} \mathrm{erg} \mathrm{s}^{-1}$. b) Models with $q_{\mathrm{e}}=10^{-5}$ : for $\Gamma_{\text {jet }}=1.5,5$; and $L_{\text {cor }}=10^{34}, 10^{37} \mathrm{erg} \mathrm{s}^{-1}$.

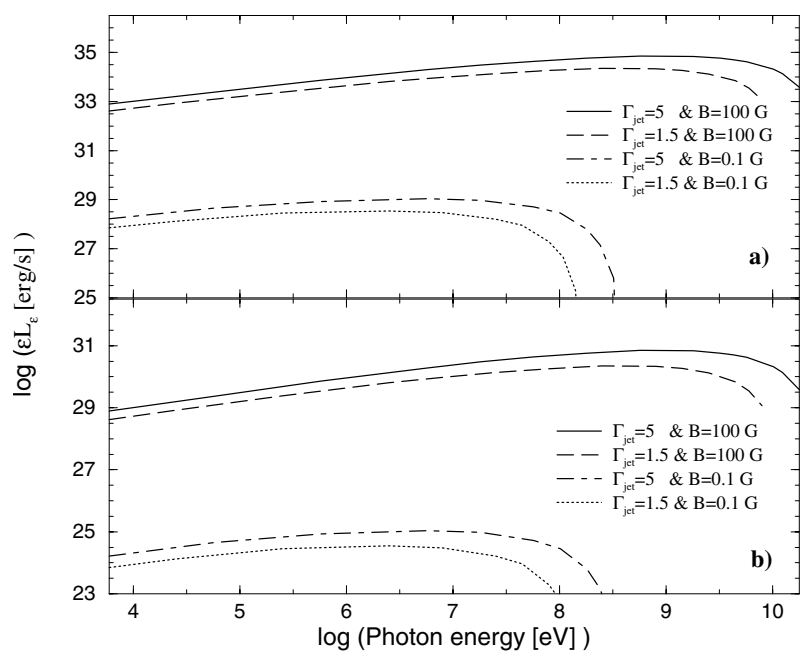

Fig. 5. High-energy SED from IC up-scattering of synchrotron photons. a) Models with $q_{\mathrm{e}}=10^{-3}$ : for $\Gamma_{\text {jet }}=1.5,5$; and $B\left(z_{0}\right)=0.1$, 100 G. b) Models with $q_{\mathrm{e}}=10^{-5}$ : for $\Gamma_{\text {jet }}=1.5,5$; and $B\left(z_{0}\right)=0.1$, $100 \mathrm{G}$.

Table 3. Range of parameters adopted for calculation of different models.

\begin{tabular}{lc}
\hline \hline Parameter (symbol) & Value \\
\hline Corona luminosity $\left(L_{\text {cor }}\right)$ & $10^{34}, 10^{37} \mathrm{erg} \mathrm{s}^{-1}$ \\
Magnetic field $\left(B\left(z_{0}\right)\right)$ & $0.1,100 \mathrm{G}$ \\
Bulk Lorentz factor of the jet $\left(\Gamma_{\text {jet }}\right)$ & $1.5,5$ \\
Disk/jet coupling constant $\left(q_{\mathrm{e}}\right)$ & $10^{-5}, 10^{-3}$ \\
\hline
\end{tabular}

\subsection{Electron energy evolution}

In this subsection, we discuss whether our parameterization is consistent with the local cooling rates of the electrons due to energy losses. This will allow us to know to what extent the evolution of the electrons is well described through the adopted parameterization, that corresponds to adiabatic expansion. If
Table 4. Parameters for a representative microquasar model.

\begin{tabular}{lc}
\hline \hline Parameter (symbol) & Value \\
\hline Corona luminosity $\left(L_{\text {cor }}\right)$ & $10^{35} \mathrm{erg} \mathrm{s}^{-1}$ \\
Magnetic field $\left(B\left(z_{0}\right)\right)$ & $10 \mathrm{G}$ \\
Maximum electron Lorentz factor $\left(\gamma_{\mathrm{emax}}\left(z_{0}\right)\right)$ & $10^{4}$ \\
Bulk Lorentz factor of the jet $\left(\Gamma_{\text {jet }}\right)$ & 2.5 \\
Disk/jet coupling constant $\left(q_{\mathrm{e}}\right)$ & $10^{-4}$ \\
\hline
\end{tabular}

Table 5. Parameters for an extreme case (microblazar).

\begin{tabular}{lc}
\hline \hline Parameter (symbol) & Value \\
\hline Corona luminosity $\left(L_{\text {cor }}\right)$ & $10^{35} \mathrm{erg} \mathrm{s}^{-1}$ \\
Magnetic field $\left(B\left(z_{0}\right)\right)$ & $10 \mathrm{G}$ \\
Maximum electron Lorentz factor $\left(\gamma_{\mathrm{emax}}\left(z_{0}\right)\right)$ & $10^{6}$ \\
Jet bulk Lorentz factor $\left(\Gamma_{\text {jet }}\right)$ & 10 \\
Viewing angle to jet's axis $\theta$ & $1^{\circ}$ \\
Disk/jet coupling constant $\left(q_{\mathrm{e}}\right)$ & $10^{-4}$ \\
\hline
\end{tabular}

Table 6. Parameters for a "realistic" case.

\begin{tabular}{lc}
\hline \hline Parameter (symbol) & Value \\
\hline Corona luminosity $\left(L_{\text {cor }}\right)$ & $3 \times 10^{32} \mathrm{erg} \mathrm{s}^{-1}$ \\
Magnetic field $\left(B\left(z_{0}\right)\right)$ & $200 \mathrm{G}$ \\
Maximum electron Lorentz factor $\left(\gamma_{\mathrm{emax}}\left(z_{0}\right)\right)$ & $10^{4}$ \\
Jet bulk Lorentz factor $\left(\Gamma_{\text {jet }}\right)$ & 1.1 \\
Viewing angle to jet's axis $\theta$ & $10^{\circ}$ \\
Disk/jet coupling constant $\left(q_{\mathrm{e}}\right)$ & $10^{-3}$ \\
\hline
\end{tabular}

radiative losses are smaller than adiabatic ones, then the parameterization is fine at least as a first order of approximation.

We have determined quantitatively the importance of the energy density of the different seed photon fields, taking into account in our computations both the internal ones (synchrotron photon and magnetic fields) and the external ones (star, disk and corona photon fields). In Table 7, we show the energy densities for the mentioned photon fields in the comoving frame at the base of the jet for the three microquasar models we have calculated (see Tables 4-6). In Fig. 9, we show the evolution of the cooling times along the jet for the adiabatic and the radiative losses. This allows us to find out which one dominates at different distances. To compute and compare the different cooling times, we have taken the electron Lorentz factor which would correspond in our model to a given value of $z$, considering the adiabatic evolution of electrons. In this plot, we show the behavior of the "realistic" and the representative cases as examples; the microblazar case is not too different.

From Fig. 9, it is clear that it is enough in the "realistic" case to account only for adiabatic losses to describe the electron energy evolution along the jet, whereas it is not sufficient for the representative case at small values of $z$. In the second situation, however, this is not a problem since we do not pretend to describe the microphysics within the jet, but only to 
Table 7. Energy densities at the base of the jet in units of $\mathrm{erg}^{\mathrm{cm}}{ }^{-3}$.

\begin{tabular}{lccccccc}
\hline \hline Model & $\begin{array}{c}\text { Jet velocity } \\
\text { (c) }\end{array}$ & Lorentz factor & $\begin{array}{c}\text { Magnetic } \\
\text { field }\end{array}$ & $\begin{array}{c}\text { Synchrotron } \\
\text { photon field }\end{array}$ & $\begin{array}{c}\text { Star } \\
\text { photon field }\end{array}$ & $\begin{array}{c}\text { Disk } \\
\text { photon field }\end{array}$ & $\begin{array}{c}\text { Corona } \\
\text { photon field }\end{array}$ \\
\hline Representative & $0.917 \mathrm{c}$ & 2.5 & 2 & 3.2 & 220 & $2.4 \times 10^{5}$ & $2.2 \times 10^{6}$ \\
Microblazar & $0.995 \mathrm{c}$ & 10 & 2 & 49.8 & $1.6 \times 10^{4}$ & 3.7 & $3.4 \times 10^{4}$ \\
"Realistic" & $0.417 \mathrm{c}$ & 1.1 & 800 & $6.3 \times 10^{4}$ & 7.6 & 87.8 & $8.2 \times 10^{4}$ \\
\hline
\end{tabular}

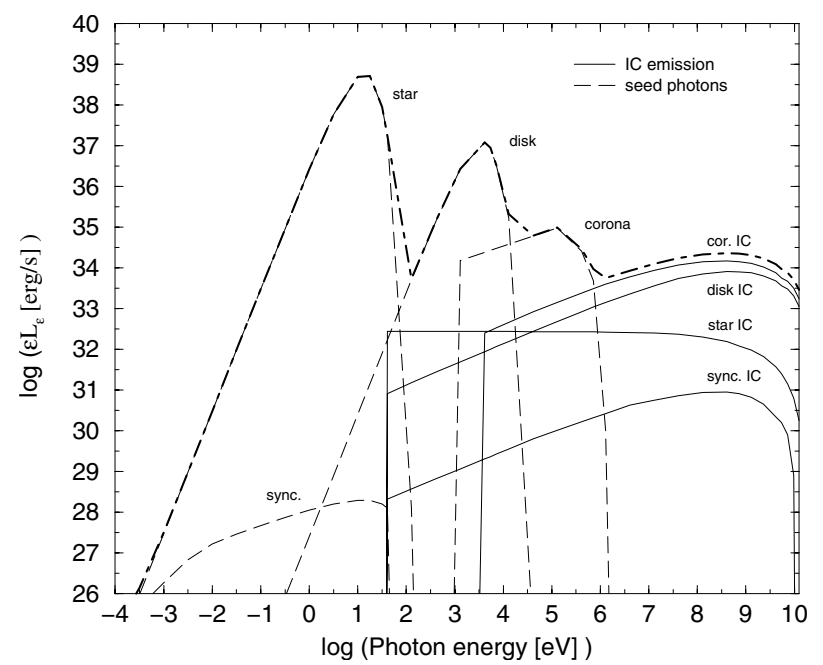

Fig. 6. Complete SED for a model with $q_{\mathrm{e}}=10^{-4}, B\left(z_{0}\right)=10 \mathrm{G}$, $L_{\mathrm{cor}}=10^{35} \mathrm{erg} \mathrm{s}^{-1}, \gamma_{\mathrm{emax}}\left(z_{0}\right)=10^{4}, \Gamma_{\mathrm{jet}}=2.5$, and a viewing angle of $10^{\circ}$. This is the representative case.

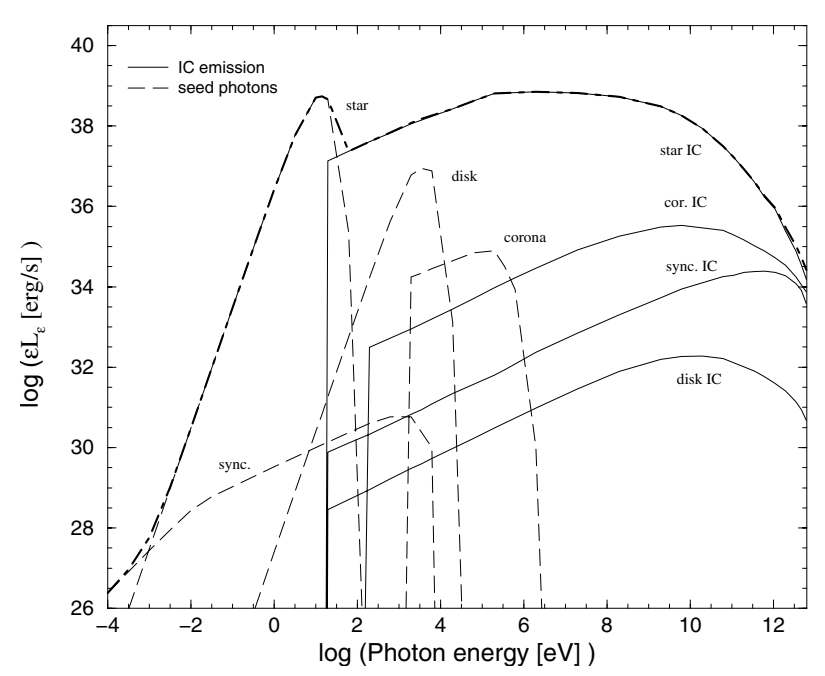

Fig. 7. SED for a model with $q_{\mathrm{e}}=10^{-4}, B\left(z_{0}\right)=10 \mathrm{G}, L_{\text {cor }}=$ $10^{35} \mathrm{erg} \mathrm{s}^{-1}, \gamma_{\mathrm{emax}}\left(z_{0}\right)=10^{6}, \Gamma_{\mathrm{jet}}=10$, and a viewing angle of $1^{\circ}$. This is the extreme case.

parametrize it in such a way that the expected emission will be observed. This parameterization could take into account reacceleration processes acting on electrons. The assumption that electrons are accelerated along the jet is natural for many jet models (see, i.e., Biermann \& Strittmater 1987). Future work will be done to implement this in a more explicit way.

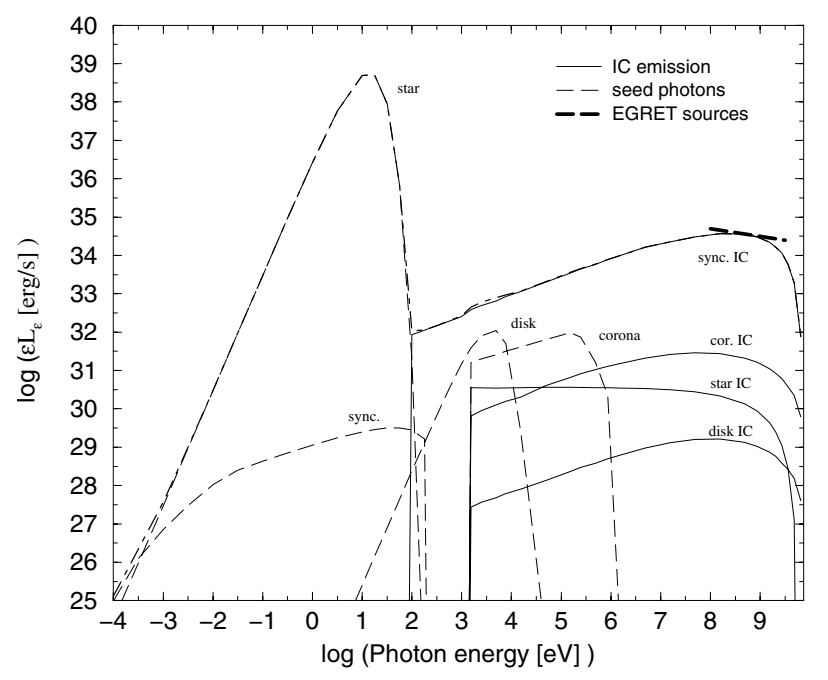

Fig. 8. SED for a model with $q_{\mathrm{e}}=10^{-3}, B\left(z_{0}\right)=200 \mathrm{G}, L_{\text {cor }}=3 \times$ $10^{32} \mathrm{erg} \mathrm{s}^{-1}, \gamma_{\mathrm{emax}}\left(z_{0}\right)=10^{4}, \Gamma_{\text {jet }}=1.1$, and a viewing angle of $10^{\circ}$. This is the "realistic" case.

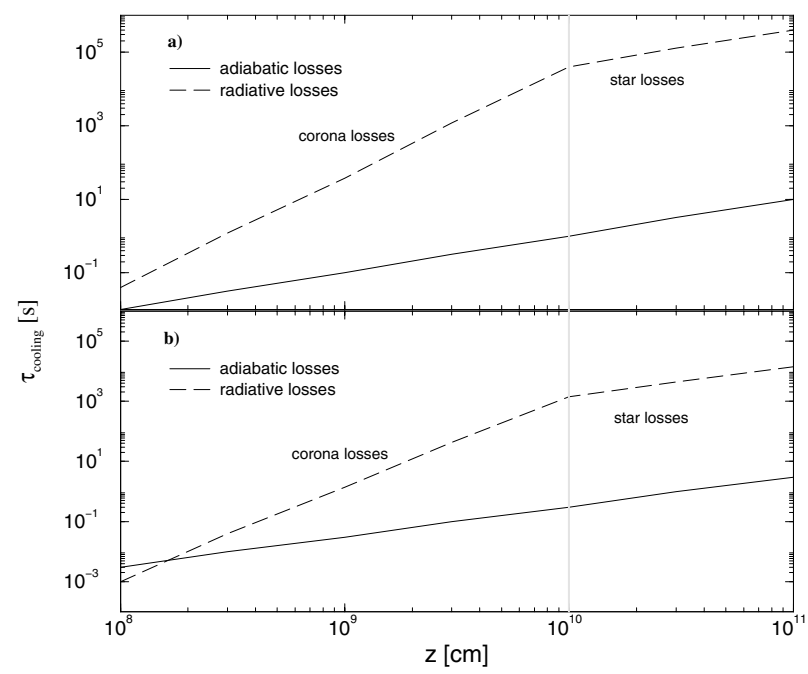

Fig. 9. Cooling time evolution with $z$ for adiabatic (solid line) and radiative (long-dashed line) losses. Two regions in the plot have been established depending on the dominant source of radiative losses, which happen to be the corona IC losses close to the compact object and the star IC losses farther away. a) "Realistic" case. b) Representative case.

\section{Comments}

We can see from Figs. 2-5 that for several models with disk/jet coupling constant $q_{\mathrm{e}}=10^{-3}$ we can get the expected luminosities in the observer's frame inferred for GRP I sources with the right photon index at energies $\sim 1 \mathrm{GeV}$, i.e. $\Gamma \sim 2$. When the 
magnetic field is strong enough $\left(B\left(z_{0}\right) \sim 100 \mathrm{G}\right)$, SSC emission alone can account for luminosities $\sim 10^{35} \mathrm{erg} \mathrm{s}^{-1}$ at $1 \mathrm{GeV}$. Models with bulk Lorentz factors $\Gamma_{\text {jet }}=1.5$ and 5 do not produce dramatically different results for a jet with a viewing angle of $\sim 10^{\circ}$ except for the case of the scattered stellar photons. In case of lower magnetic fields, the IC scattering upon external fields clearly dominates. For instance, when $B\left(z_{0}\right)=0.1 \mathrm{G}$, the IC component upon stellar seed photons is more than 5 orders of magnitude greater than the SSC emission at $100 \mathrm{MeV}$. For a similar value of $q_{\mathrm{e}}$ and a $\Gamma_{\text {jet }}=5$, the stellar IC component usually dominates over the disk and corona components. Only in the case of a powerful corona contribution $\left(L_{\mathrm{cor}} \sim 10^{37} \mathrm{erg} \mathrm{s}^{-1}\right)$ can the latter surpass the IC emission produced in the stellar field. The contribution arising from interactions with disk photons reaches values $\sim 10^{34} \mathrm{erg} \mathrm{s}^{-1}$ only for energetic jets $\left(q_{\mathrm{e}}=10^{-3}\right)$.

Models with light leptonic jets $\left(q_{\mathrm{e}}=10^{-5}\right)$ can produce significant gamma-ray sources $\left(\sim 10^{34} \mathrm{erg} \mathrm{s}^{-1}\right.$ at $\left.100 \mathrm{MeV}\right)$ when a strong corona is present (see Fig. 4). In these models, the spectrum tends to be a bit harder than in the case of stellar photons, with our current set of assumptions (value of $\Gamma_{\text {cor }}$, etc).

Comparing Figs. 6 and 7 it is possible to see the differences between a "mild" microquasar, with $\Gamma_{\text {jet }}=2.5$, a viewing angle of $\theta=10^{\circ}$ and $q=10^{-4}$, and a more "extreme" case with $\Gamma_{\text {jet }}=10$ and $\theta=1^{\circ}$, i.e. a microblazar. Since we have assumed in both cases a magnetic field of $B\left(z_{0}\right)=10 \mathrm{G}$, external IC scattering always dominates over SSC. In the "mild" microquasar the emission at $\mathrm{MeV}-\mathrm{GeV}$ energies is mainly due to upscattering of coronal photons whereas, in the extreme case, the IC emission in the stellar field exceeds by far the other contributions. The hard X-ray counterpart can be important, even beyond the cutoff for the corona (at $\sim 150 \mathrm{keV}$ ). Hence, INTEGRAL observations can be useful to unmask candidates that are obscured at optical wavelength (see Combi et al. 2004 for a recent study in this direction).

An interesting feature of the extreme case is that the gamma-ray emission with a hard spectrum $\Gamma \sim 2$ extends up to tens of $\mathrm{GeV}$. At $\mathrm{TeV}$ energies, the spectrum becomes very soft due to the Klein-Nishina effect. This type of sources should be detectable with modern Imaging Cherenkov Telescopes like HESS and MAGIC. Even systems with low-mass companions and weak coronas might be detectable since the SSC component has luminosities of $\sim 10^{35} \mathrm{erg} \mathrm{s}^{-1}$.

In the case we have called "realistic" (Fig. 8), the highenergy emission is dominated by SSC. We emphasize that this case is more realistic than the previous ones only in the sense that it fits well the broadband spectra of known microquasars that are suspected to be high-energy gamma-ray sources. A basic feature of these objects is their weak X-ray luminosity, a feature that is shared by most of the unidentified EGRET sources.

We now comment on the main differences between the models presented here and those already published by Georganopoulos et al. (2002) and Bosch-Ramon \& Paredes (2004a). The first authors consider just the interaction of a homogeneous jet with disk and stellar photon fields. They work in the Thomson regime with the aim of producing the hard X-ray emission observed in objects similar to Cygnus X-1 as a result of the IC contributions. No magnetic field is considered and adiabatic losses are not taken into account. On the contrary, we adopt a thermal Comptonization field at hard X-rays, possibly originating in a corona around the black hole (e.g. Zdziarski et al. 2003), as suggested by the observation of a Compton reflection feature and an iron $\mathrm{K} \alpha$ line in some sources (for an alternative interpretation, see Markoff et al. 2003b). In this sense, our treatment is more similar to that of Romero et al. (2002). However, we include the effects of the magnetic field as well as a more sophisticated parameterization of the jet. SSC emission, in fact, seems to dominate for some reasonable choices of the parameters. In addition, we go to much higher energies, taking into account the Klein-Nishina effect. Regarding the Bosch-Ramon \& Paredes (2004a) model, here we introduce several improvements, from the corona effect up to a $z$ dependence of the magnetic field and other parameters.

\section{Discussion}

Variability in the gamma-ray emission of microquasars on timescales from days to months can be caused by both changes in factors external to the jet or in the jet itself. The winds of the companion star can change inducing variations in the accretion rate (Reig et al. 2003; McSwain et al. 2004). Also, if the orbit of the compact object is not completely circularized, periodic changes in the IC flux from the stellar photon field and in the accretion rate can be expected. These changes would vary, in turn, the power carried by the jet (through changes in $q_{\mathrm{e}}$ ), the emission of the jet, and the emission of the thermal plasma around the black hole. Precession of the accretion disk (with timescales of months, e.g. Brocksopp et al. 1999) might also result in a time-modulation of the corresponding soft X-ray field. Precession of the jet itself can, additionally, produce strong variability due to the variation of the Doppler factor (Kaufman Bernadó et al. 2002). All these contributions might result in a complex lightcurve, with several associated timescales.

In sources where the jet is steady and a transient feature associated with the low-hard state, its disappearance in the high-soft state would lead, of course, to the suppression of the gamma-ray emission. This should be an additional source of variability. This transition, however, seems not to occur in microquasars with persistent jets like LS 5039.

Besides medium and long-term variations, the presence of shocks in the jets can introduce very rapid changes in the gamma-ray flux. Relativistic shocks are the natural result of sudden changes in the injection rate of plasma in the jets. These shocks propagate downstream increasing the energy of the particles in the fluid and amplifying the magnetic field. When the shock finds a small feature in the underlying jet (e.g. an inhomogeneity in the particle density or a bend in the flow direction, see Romero 1996) a very rapid variation in the high-energy flux can occur. The typical timescale for these events will be determined by the time it takes the shock to move through the feature. Assuming that the feature has a size of the order of the jet radius, this gives:

$t_{\mathrm{var}} \sim \frac{R_{\mathrm{jet}}}{D_{\mathrm{s}} v_{\mathrm{s}}}$ 
where $D_{\mathrm{s}}$ is the Doppler factor of the shock and $v_{\mathrm{S}}$ its velocity. For $R_{\text {jet }} \sim 10^{7} \mathrm{~cm}$, and a relativistic shock with $D_{\mathrm{s}} \sim 5$ and $v_{\mathrm{s}} \sim$ $c$, we have $t_{\mathrm{var}} \sim 10^{-4} \mathrm{~s}$, so this would be a very rapid flickering superposed on the longer variability.

Microquasars are not, of course, the only kind of galactic object that might display variable gamma-ray emission. Other alternatives include early-type binaries (Benaglia \& Romero 2003), accreting neutron stars (Romero et al. 2001), pulsar wind nebulae (Roberts et al. 2002) and exotic objects (Punsly et al. 2000). However, microquasars are perhaps the most attractive candidates to explain a significant fraction of the variable GRP I sources because of the presence of relativistic jets in these objects, as well as the external photon fields provided by the companion star and the accreting matter. GRP II sources might also be associated with old low-mass microquasars if the jets of these objects have magnetic fields strong enough to make SSC the primary source of radiation (Kaufman Bernadó et al. 2004).

\section{Conclusion}

We have shown that the variable gamma-ray sources found on the galactic plane have some common features that make it reasonable to consider them as a distinctive group of GRP I sources. We have suggested that these sources might be microquasars with high-mass stellar companions and we have developed some detailed models to explain the gamma-ray production in this type of objects. In particular, we have considered inhomogeneous jet models where gamma-rays are the result of inverse Compton interactions of leptons in the jet with locally produced synchrotron photons as well as external photon fields. We have calculated the emission resulting from the upscattering of disk, coronal, and stellar photons, incorporating a full Klein-Nishina calculation and the effect of losses in the particle spectrum. We have shown that a wide variety of spectral energy distributions at high energies can be obtained from different and reasonable combinations of the physical parameters like magnetic field, jet power, coronal and disk luminosities, etc. It seems clear that the microquasar phenomenon can be naturally extended up to the highest energies and that we can expect these objects to manifest themselves as a distinctive group of gamma-ray sources that might be detectable with satellite-borne instruments like those to be carried by AGILE and GLAST, and even by ground-based Cherenkov telescopes like HESS and MAGIC.

Acknowledgements. We thank Marina Kaufman Bernadó, Felix Aharonian and Peter Biermann for useful discussions on microquasars. We also thank an anonymous referee for constructive comments on the manuscript. V.B-R. and J.M.P. acknowledge partial support by DGI of the Ministerio de Ciencia y Tecnología (Spain) under grant AYA-2001-3092, as well as additional support from the European Regional Development Fund (ERDF/FEDER). During this work, V.B-R has been supported by the DGI of the Ministerio de Ciencia y Tecnología (Spain) under the fellowship FP-2001-2699. G.E.R. has been supported by Fundación Antorchas and the Argentine agencies ANPCyT and CONICET (PIP 0438/98).
This project benefited from an international cooperation grant funded by Fundación Antorchas.

\section{References}

Atoyan, A. M., \& Aharonian, F. A. 1999, MNRAS, 302, 253

Benaglia, P., \& Romero, G. E. 2003, A\&A, 399, 1121

Bhattacharya, D., Akyüz, A., Miyagi, T., Samimi, J., \& Zych, A. 2003, A\&A, 404, 163

Biermann, P. L., \& Strittmatter, P. A. 1987, ApJ, 322, 643

Blumenthal, G. R., \& Gould, R. J. 1970, Rev. Mod. Phys., 42, 237

Bosch-Ramon, V., \& Paredes, J. M. 2004a, A\&A, 417, 1075

Bosch-Ramon, V., \& Paredes, J. M. 2004b, A\&A, 425, 1069

Brocksopp, C., Fender, R. P., Larimov, V., et al. 1999, MNRAS, 309, 1063

Combi, J. A., Ribó, M., Mirabel, I. F., \& Sugizaki, M. 2004, A\&A, 422,1031

Dermer, C. D., Schlickeiser, R., \& Mastichiadis, A. 1992, A\&A, 256, L27

Dermer, C. D., \& Schlickeiser, R. 2002, ApJ, 575, 667

Falcke, H., \& Biermann, P. L. 1995, A\&A, 293, 665

Falcke, H., \& Biermann, P. L. 1999, A\&A, 342, 49

Gehrels, N., Macomb, D. J., Bertsch, D. L., Thompson, D. J., \& Hartman, R. C. 2000, Nature, 404, 363

Georganopoulos, M., Aharonian, F. A., \& Kirk, J. G. 2002, A\&A, 388, L25

Ghisellini, G., Maraschi, L., \& Treves, A. 1985, A\&A, 146, 204

Grenier, I. A. 1995, Adv. Space Res., 15, 573

Grenier, I. A. 2001, in The Nature of Unindentified Galactic High-Energy Gamma-Ray Sources, ed. A. Carraminana, O. Reimer, \& D. Thompson (Dordrecht: Kluwer Academic Publishers), 51

Grenier, I. A. 2004, in Cosmic Gamma-Ray Sources, ed. K. S. Cheng, \& G. E. Romero (Dordrecht: Kluwer Academic Publishers), in press

Grimm, H. J., Gilfanov, M., \& Sunyaev, R. 2002, A\&A, 391, 923

Hartman, R. C., Bertsch, D. L., Bloom, S. D., et al. 1999, ApJS, 123, 79

Kaufman Bernadó, M. M., Romero, G. E., \& Mirabel, I. F. 2002, A\&A, 385, L10

Kaufman Bernadó, M. M., et al. 2004, in preparation

Maccarone, T. J. 2002, MNRAS, 336, 1371

Markoff, S., Falcke, H., \& Fender, R. 2001, A\&A, 372, L25

Markoff, S., Nowak, M., Corbel, S., et al. 2003, A\&A, 397, 645

Markoff, S., Nowak, M., Corbel, S., et al. 2003b, New Astron. Rev., 47, 491

Massi, M., Ribó, M., Paredes, J. M., et al. 2004, A\&A, 414, L1

McClintock, J. E., \& Remillard, R. A. 2004, Chapter 4 in Compact Stellar X-ray Sources, ed. W. H. G. Lewin, \& M. van der Klis (Cambridge University Press), in press [arXiv: astro-ph/0306213]

McSwain, M. V., Gies, D. R., Huang, W., et al. 2004, ApJ, 600, 927

Mirabel, I. F., \& Rodríguez, L. F. 1999, ARA\&A, 37, 409

Mirabel, I. F., Rodrigues, I., \& Liu, Q. Z. 2004, A\&A, 422, L29

Miyagi, T., \& Bhattacharya, D. 2004, personal communication

Nolan, P. L., Tompkins, W. F., Grenier, I. A., \& Michelson, P. F. 2003, ApJ, 597, 615

Pacholczyk, A. G. 1970, Radio Astrophysics (San Francisco, CA: Freeman)

Paredes, J. M., Martí, J., Ribó, M., \& Massi, M. 2000, Science, 288, 2340 
Paredes, J. M., Ribó, M., Ros, E., et al. 2002, A\&A, 393, L99

Poutanen, J. 1998, in Theory of Black Hole Accretion Disks, ed. M. Abramowicz et al. (Cambridge: Cambridge University Press), 100

Punsly, B., Romero, G. E., Torres, D. F., \& Combi, J. A. 2000, A\&A, 364, 552

Reig, P., Ribó, M., Paredes, J. M., \& Martí, J. 2003, A\&A, 405, 285

Reimer, O. 2001, in The Nature of Unindentified Galactic High-Energy Gamma-Ray Sources, ed. A. Carraminana, O. Reimer, \& D. Thompson (Dordrecht: Kluwer Academic Publishers), 17

Ribó, M., Paredes, J. M., Romero, G. E., et al. 2002, A\&A, 384, 954

Roberts, M. S. E., Gaensler, B. M., \& Romani, R. W. 2002, in Neutron Stars in Supernova Remnants, ed. Patrick O. Slane, \& Bryan M. Gaensler (San Francisco: ASP), ASP Conf. Ser., 271, 213

Romero, G. E. 1996, A\&A, 313, 759

Romero, G. E., Benaglia, P., \& Torres, D. F. 1999, A\&A, 348, 868
Romero, G. E. 2001, in The Nature of Unindentified Galactic HighEnergy Gamma-Ray Sources, ed. A. Carraminana, O. Reimer, \& D. Thompson (Dordrecht: Kluwer Academic Publishers), 65

Romero, G. E., Kaufman Bernadó, M. M., Combi, J. A., \& Torres, D. F. 2001, A\&A, 376, 599

Romero, G. E., Kaufman Bernadó, M. M., \& Mirabel, I. F. 2002, A\&A, 393, L61

Romero, G. E., Torres, D. F., Kaufman Bernadó, M. M., \& Mirabel, I. F. 2003, A\&A, 410, L1

Romero, G. E., Grenier, I. A., Kaufman Bernadó, M. M., Mirabel, I. F., \& Torres, D. F. 2004, ESA-SP, in press [arXiv: astro-ph/0402285]

Torres, D. F., Romero, G. E., Combi, J. A., et al. 2001, A\&A, 370, 468

Zdziarski, A. A., Lubinski, P., Gilfanov, M., \& Revnivtsev, M. 2003, MNRAS, 342, 355 